
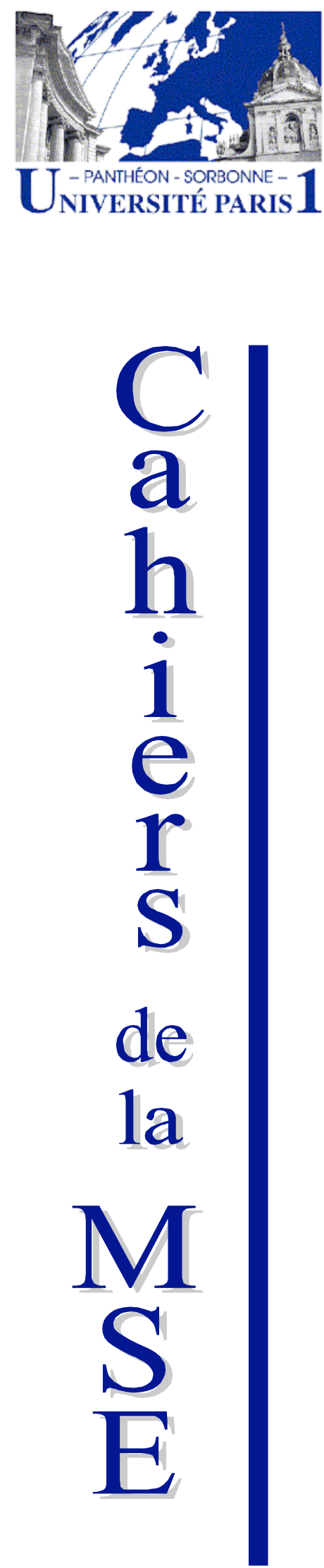

When does a developing country use new technologies?

Olivier BRUNO, GREDEG

Cuong Le VAn, CeRmsem

Benoît MASQUIN, GREDEG

2005.93 


\title{
When Does a Developing Country Use New Technologies?*
}

\author{
Olivier Bruno ${ }^{\dagger}$ Cuong Le Van $\ddagger$ and Benoît Masquin ${ }^{\S}$
}

July 6, 2005

\begin{abstract}
We develop a model of optimal pattern of economic development that is first rooted in physical capital accumulation and then in technical progress. We study an economy where capital accumulation and innovative activity take place within a two sector model. The first sector produces a consumption good using physical capital and non skilled labor. Technological progress in the consumption sector is driven by the research activity that takes place in the second sector. Research activity which produces new technologies requires technological capital and skilled labor. New technologies induce an endogenous increase of the Total Factor Productivity of the consumption sector. Physical and technological capital are not substitutable while skilled and non skilled labor may be substitutable.

We show that under conditions on the adoption process of new technologies, the optimal strategy for a developing country consist in accumulating physical capital first; postponing the importation of technological capital to the second stage of development. This result is due to a threshold effect from which new technologies begin to have an impact on the productivity of the consumption sector. However, we show that once a certain level of wealth is reached, it becomes optimal for the economy to import technological capital to produce new technologies.
\end{abstract}

${ }^{*}$ The authors would like to thank the participants to the seminar of GREDEG, especially Richard Arena, Flora Bellone, Jean-Luc Gaffard and Jacques Ravix, and also the participants to a seminar in European University Institute

${ }^{\dagger}$ GREDEG, UNSA

${ }^{\ddagger}$ GREDEG, CERMSEM, CNRS, UNSA, University Paris 1

$\S_{\text {GREDEG, UNSA }}$ 


\section{Introduction}

It is still rather unclear whether capital accumulation matters relatively more than technological progress in the growth of developing countries.

The Krugman - Solow controversy about the Asian Miracle illustrates particularly well this divergence between those who think that capital accumulation is an unimportant part of the growth process and those who think that it is a fundamental factor of growth for developing countries.

According to the traditional growth theories, poorer countries grow faster than richer ones during their first stage of development. This result is rooted in the assumption of decreasing returns to scale on capital accumulation which induce a catching-up process compatible with conditional convergence (Cass [1965]). However, cross-countries empirical studies show that development patterns differ considerably between countries in the long run (Barro\&Sala-i-Martin [1995], Barro [1997]). These differences can be explained within a model of capital accumulation with convex - concave technology. In such a framework, Dechert and Nishimura [1983] prove the existence of threshold effect with poverty traps explaining alternatively "growth collapses" or taking-off. In all those models, the rate of growth is exogenous. Alternatively, other models propose several ways for endogeneizing the rate of growth: they state that growth patterns are influenced by educational resources, innovation processes and technical differences between countries. Therefore, developing countries initially follow divergent paths of growth corresponding to international differences in factor endowments and in patterns of government intervention. However, this point is in contradiction with most of the empirical studies stressing the role played by capital accumulation in early stages of growth. ${ }^{1}$

According to these empirical studies, it seems that the respective weight of capital accumulation and technical progress which contributes to explain the rate of growth of a country depends on its level of development. The higher the level of development the higher the weight of technical progress, and the smaller the relative share of capital accumulation (Kim and Lau [1994])

Even if this idea seems to be spreading in the profession, it seems there is still no model explaining the optimal switch of a country from the first stage to the second stage of development. In this paper, we present a model

\footnotetext{
${ }^{1}$ Notice that threshold effect is also used by Le Van and Saglam [2004] who show that a developing country can retain to invest in technology if the initial knowledge of the country and the quality of knowledge technology are low or if the level of fixed costs of the production technology are high.
} 
which aims at explaining this switch. We show that the optimal pattern of growth of a developing country is initially determined by physical capital accumulation. Later, the technological progress will appear when the country has reached a certain level of development. Capital accumulation and innovative activity take place within a two sector growth model. The first sector produces a consumption good using physical capital and non skilled labor according to a Cobb-Douglas production function. Technological progress in the consumption sector is driven by the research activity that takes place in the second sector. Research activity which produces new technologies requires technological capital and skilled labor along the line of a Cobb-Douglas production function. When new technologies produced by the research activity are used in the consumption sector they induce an endogenous increase of the Total Factor Productivity. The two kinds of capital are not substitutable while skilled and non skilled labors may be substitutable.

We suppose that technological capital, used by the research activity, is not produced within the economy. The domestic economy must purchase it in the international market at a given price. Consequently, the consumption good can be consumed, invested as physical capital or exported in order to import technological capital. The price of the consumption good is given by the international market and is used as numeraire in our economy. Finally, we assume that physical capital is less costly than technological capital.

Our model exhibits first decreasing returns and then increasing returns, and from this point of view it differs from Dechert and Nishimura [1983] seminal model which is convex-concave.

We show that under conditions on the adoption process of new technologies, the optimal strategy for a developing country consist in accumulating physical capital first; postponing the importation of technological capital to the second stage of development. thus, all resources of the economy are devoted to consumption or investment in the physical capital and there is no research activity. The growth process is initially driven by capital accumulation using concave technology. This result is due to a threshold effect from which new technologies begin to have an impact on the productivity of the consumption sector. Indeed, it is necessary to have a minimum amount of adoption of new technologies in order for them to be efficient. This may be due to technological complementarity between new technologies or to a network effect (Ciccone and Matsuyama (1999)). Threshold is related to three factors: the amount of available human capital, the price of technological capital and the initial income of the economy. For given values of these factors, we show that there is a time period after which it is optimal 
for the economy to import technological capital in order to produce new technologies. From that date onwards, research activity generates an endogenous technical change and the economy follows an optimal endogenous growth path with increasing returns to scale technology. Our model exhibits an optimal pattern of economic development that is first rooted in physical capital accumulation and then in technical progress. As a consequence of the existence of the threshold, a country may never take off and may converge towards a traditional steady state. This explains that the international convergence or divergence of income levels tightly depends on the value of the threshold effect.

The initial value of human capital plays an essential role in the process we have just described. The higher this value, the sooner research activity and endogenous growth will take place. This result is in accordance with the empirical study of Benhabib and Spiegel [1994] showing that growth is related to the initial level of human capital and not to the accumulation of human capital.

In the last part of the model (Section 5) we relax the assumption of non substitutability between the two kinds of labor. We allow high-skilled workers to work in the production sector. We show that the optimal endogenous growth path may be compatible with an underutilization of high-skilled labor in the research activity. However, if the number of high-skilled workers is low relatively to the low-skilled workers then, after a certain time, the technological sector will full employ high-skilled workers.

Our paper is organized as follows. We set the model in Section 2. We first study the one period economy in Section 3. Section 4 deals with the infinitely lived optimal growth model with non substitutable labor force. In Section 5, we allow high skilled workers shift towards consumption sector but low skilled workers cannot join the innovative sector. Section 6 gathers the main results of our paper.

\section{The Structure of the Economy}

We consider a developping country which produces a consumption good $Y_{d}$ with physical captial $K_{d}$ and low-skilled labor $L_{d}$. The consumption sector may use a quantity of new technologies $Y_{e}$ to increase its Total Factor Productivity. We have:

$$
Y_{d}=\phi\left(Y_{e}\right) K_{d}^{\alpha_{d}} L_{d}^{1-\alpha_{d}}
$$

where $\alpha_{d} \in(0,1)$ and $\phi$ is a non decreasing function which verifies $\phi(0)=$ $x_{0}>0$. 
The amount of new technologies $Y_{e}$ may be produced through a CobbDouglas function using technological capital $K_{e}$ and high-skilled labor $L_{e}$. We have:

$$
Y_{e}=A_{e} K_{e}^{\alpha_{e}} L_{e}^{1-\alpha_{e}} .
$$

where $\alpha_{e} \in(0,1)$ and $A_{e}$ is the total productivity.

The economy cannot produce technological capital whereas physical capital and consumption good are homogenous. It exports consumption good $Y_{d}$ in order to import technological capital. We use domestic consumption good as numeraire. Prices are respectively $\lambda>1$ for technological capital and 1 for consumption good and physical capital.

Let $S$ be the value of the initial wealth of the country in terms of consumption good. $S$ could take the form of a development aid granted to the developing economy by foreign countries. It can be either used for physical capital accumulation or for the importation of technological capital. The budget constraint of the economy is:

$$
K_{d}+\lambda K_{e}=S
$$

\section{The Single Period Model}

The social planner maximizes the following program

$$
\max _{c, K_{e}, K_{d}, L_{e}, L_{d}}\{u(c)\}
$$

under the constraints:

$$
\begin{gathered}
c=Y_{d} \\
Y_{d}=\phi\left(Y_{e}\right) K_{d}^{\alpha_{d}} L_{d}^{1-\alpha_{d}} \\
Y_{e}=A_{e} K_{e}^{\alpha_{e}} L_{e}^{1-\alpha_{e}} \\
K_{d}+\lambda K_{e}=S \\
L_{d} \leq L_{d}^{*} \\
L_{e} \leq L_{e}^{*} h
\end{gathered}
$$

By assumption, the function $u$ is strictly increasing. Thus, maximising agents' utility is equivalent to maximize the quantity of consumption goods. In a single period model there will be no saving or investment (equation 
1). In (5) and (6), $L_{d}^{*}$ and $L_{e}^{*}$ are exogenous supplies of low-skilled and high-skilled workers. These inequalities state there is no possible transfer between high-skilled and low-skilled workers. We suppose the human capital for high-skilled workers is measured by the number $h \geq 1$.

Let $\theta=\frac{\lambda K_{e}}{S}$. Then (4) can be re-written

$$
K_{d}=(1-\theta) S, \lambda K_{e}=\theta S
$$

Since at the optimum, $L_{e}=L_{e}^{*} h, L_{d}=L_{d}^{*}$, the social planner's problem turns out to be

$$
\max _{\theta \in[0,1]} \phi\left(\frac{A_{e} h^{1-\alpha_{e}} L_{e}^{*^{1-\alpha_{e}}}}{\lambda^{\alpha_{e}}} \theta^{\alpha_{e}} S^{\alpha_{e}}\right)(1-\theta)^{\alpha_{d}} S^{\alpha_{d}} L_{d}^{*^{1-\alpha_{d}}}
$$

Let $r_{e}=\frac{A_{e}}{\lambda^{\alpha_{e}}} L_{e}^{*^{1-\alpha_{e}}} h^{1-\alpha_{e}}$ and $\psi\left(r_{e}, S, \theta\right)=\phi\left(r_{e} \theta^{\alpha_{e}} S^{\alpha_{e}}\right)(1-\theta)^{\alpha_{d}} L_{d}^{*^{1-\alpha_{d}}}$ Solving the previous problem becomes equivalent to solve

$$
\max _{0 \leq \theta \leq 1} \psi\left(r_{e}, S, \theta\right)
$$

Since the function $\psi$ is continuous in $\theta$, there will always be an optimal solution. Let be

$$
G\left(r_{e}, S\right)=\operatorname{Argmax}\left\{\psi\left(r_{e}, S, \theta\right): \theta \in[0,1]\right\}
$$

and $F\left(r_{e}, S\right)=\max \left\{\psi\left(r_{e}, S, \theta\right): \theta \in[0,1]\right\}$. From the Maximum Theorem, $F$ is continuous, and the maximum output of the consumption sector will be $H\left(r_{e}, S\right)=F\left(r_{e}, S\right) S^{\alpha_{d}}$.

In this program the function $\phi$ is important because it represents the way the research output impacts the economy. Two alternative situations are possible. On the one hand, new technologies may have an immediate impact on TFP. In that case, there is no adoption effect and it is always efficient to use the technological capital. On the other hand, we can assume technological complementarity between new technologies. In that case, a minimum level of adoption of new technologies is necessary in order for them to impact the economy. In the case of adoption effect, the developing country must be sufficiently abundant in resources or human capital in order to take off by buying technological capital. 


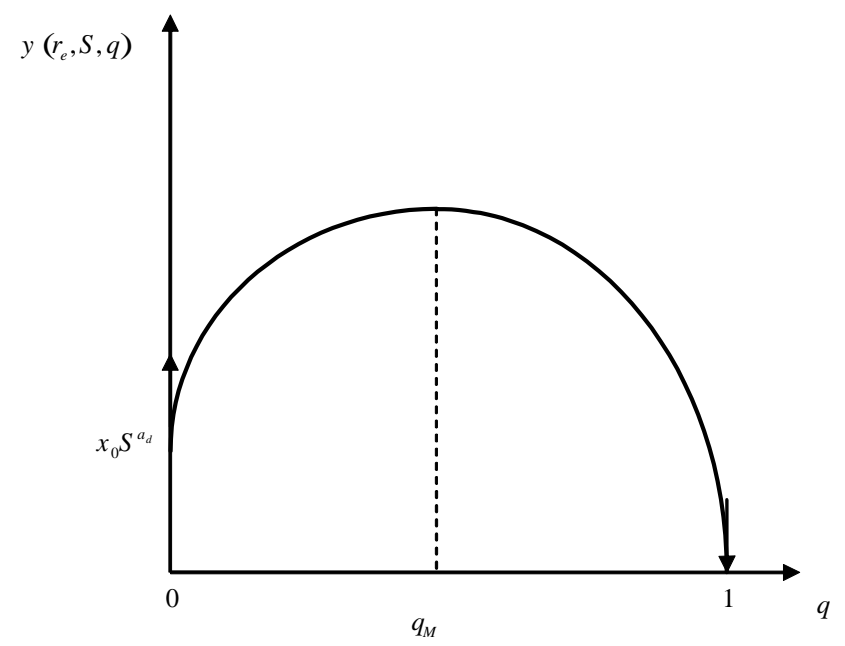

Figure 1:

\subsection{Case 1: No adoption effect of new technologies}

Proposition 1 Assume $\phi$ continuously differentiable, strictly increasing and $\phi^{\prime}(0)>0$. Then $G\left(r_{e}, S\right)$ is not empty. Moreover, $\left.G\left(r_{e}, S\right) \subset\right] 0,1[$

Proof. It's obvious that the function $\psi$ is continuouly differentiable in ]0, $1\left[\right.$. It satisfies $\psi_{\theta}^{\prime}\left(r_{e}, S, 0\right)=+\infty$ and $\psi_{\theta}^{\prime}\left(r_{e}, S, 1\right)=-\infty$. Hence, there exist $\theta_{1}$ with $\psi\left(r_{e}, S, \theta_{1}\right)>\psi\left(r_{e}, S, 0\right)$ and $\theta_{2}$ with $\psi\left(r_{e}, S, \theta_{2}\right)>\psi\left(r_{e}, S, 1\right)$. Therefore, the maximizer $\theta_{M}$ must be included in $] 0,1[$.

Figure 1 sketches $\psi\left(r_{e}, S, \theta\right)$ with $\phi$ strictly concave. In that case, the developing economy will devote an equilibrium proportion $\theta_{M}$ of its initial wealth to buy technological capital. A share of new technologies is still produced in the economy.

\subsection{Case 2: Existence of an adoption effect of new technolo- gies}

In the previous case we suppose that any rise in the quantity of technological capital, even very small, could have a direct effect on TFP of consumption 


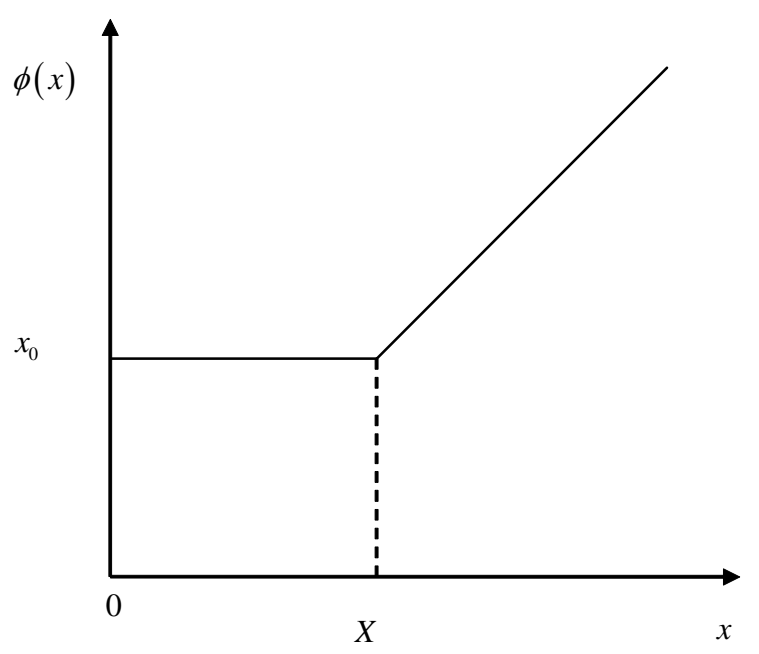

Figure 2:

sector. To analyse the impact of the adoption of new technologies, we assume there is a threshold effect from which new technologies begin to impact positively the TFP. If level of the research activity is not sufficient, it will be useless for the consumption sector.

Assume that the function $\phi$ is not strictly increasing but is supposed to have the following form:

$$
\phi(x)=\left\{\begin{array}{c}
x_{0}, \forall x \leq X \\
x_{0}+\gamma(x), \forall x \geq X
\end{array}\right\}
$$

with $x=r_{e} S^{\alpha_{e}} \theta^{\alpha_{e}}$ and $\gamma$ continuously differentiable, $\gamma^{\prime}>0, \gamma(X)=0$.

Figure 2 sketches $\phi$ when $\gamma(x)=a x, a>0$.

The technological capital is useless since $r_{e} S^{\alpha_{e}} \leq X$.

In the case $r_{e} S^{\alpha_{e}}>X$, there is a $\bar{\theta}$ that satisfies $r_{e} S^{\alpha_{e}} \bar{\theta}=X$

We can prove that if $S$ is high enough, it will be efficient to use the technological capital and produce new technologies, and when $S$ is small, it is not efficient to import technological capital.

Proposition 2 (i) If $S \leq\left(\frac{X}{r_{e}}\right)^{\frac{1}{\alpha_{e}}}$ then $G\left(r_{e}, S\right)=\{0\}$.

(ii) there exists $\hat{S}$ such that: $\left.S>\hat{S} \Rightarrow G\left(r_{e}, S\right) \subset\right] 0,1[$. 
Proof. (i) If $S \leq\left(\frac{X}{r_{e}}\right)^{\frac{1}{\alpha_{e}}}$, then for any $\theta \in[0,1]$, we have $r_{e} S^{\alpha_{e}} \theta^{\alpha_{e}} \leq X$, and hence $\psi(r, S, \theta)=x_{0}(1-\theta)^{\alpha_{d}} L_{d}^{*^{1-\alpha_{d}}}$. Obviously, the maximizer is unique and equals 0 .

(ii) Observe that $\forall S \geq 0, F\left(r_{e}, S\right) \geq x_{0} L_{d}^{*^{1-\alpha_{d}}}$. Let $S_{0}>\left(\frac{X}{r_{e}}\right)^{\frac{1}{\alpha_{e}}}$, and $\bar{\theta}\left(S_{0}\right)$ satisfies $r_{e} S_{0}^{\alpha_{e}} \bar{\theta}\left(S_{0}\right)^{\alpha_{e}}=X$. Let $\left.\hat{\theta} \in\right] \bar{\theta}\left(S_{0}\right), 1[$. When $S$ increases, $\bar{\theta}(S)$ decreases and $\hat{\theta}$ will be in $] \bar{\theta}(S), 1\left[\right.$. We have $\psi\left(r_{e}, S, \hat{\theta}\right) \rightarrow+\infty$ when $S \rightarrow+\infty$. Hence, for $S$ large enough, say, greater than some $\hat{S}$, then $\max _{\theta}\left\{\psi\left(r_{e}, S, \theta\right)\right\}>x_{0} L_{d}^{*^{1-\alpha_{d}}}$. That implies $0 \notin G\left(r_{e}, S\right)$. Since $\psi\left(r_{e}, S, 1\right)=$ 0 , we have $1 \notin G\left(r_{e}, S\right)$. The proof is complete.

Comment Observe that the larger $r_{e}$ is the higher the opportunity for the country to use new technology. Therefore, if the number of high-skilled workers is large, or if their human capital is high, or if the price of new technology is low, or if the productivity of the new technology production function is important, then the country will use new technology more rapidly.

Finally, we want to prove there exists a critical value $S^{c}$, i.e., if $S<S^{c}$ then $G\left(r_{e}, S\right)=\{0\}$ and if $S>S^{c}$ then $\left.G\left(r_{e}, S\right) \subset\right] 0,1[$. In that case, the country will import technological capital and produce new technologies as soon as its wealth $S$ is higher than the critical value $S^{c}$. Figure 3 illustrates that point.

Let $B=\left\{S \geq 0: F\left(r_{e}, S\right)=x_{0} L_{d}^{*^{1-\alpha_{d}}}\right\}$.

Lemma $3 B$ is non empty and compact.

Proof. (i) B is not empty: obviously, $0 \in B$.

(ii) $B$ is closed because the function $F$ is continuous.

(iii) To prove that $B$ is bounded take a sequence $S_{n}$ converging to $+\infty$. Fix some $\theta \in] 0,1\left[\right.$. For $n$ large enough, $\bar{\theta}\left(S_{n}\right)<\theta<1$. Then $\psi\left(r_{e}, S_{n}, \theta\right)$ converges to $+\infty$. This implies $F\left(r_{e}, S_{n}\right)>x_{0} L_{d}^{*^{1-\alpha} d}$ for any $n$ sufficiently large. That contradicts $S_{n} \in B$.

Proposition 4 Let $S^{c}=\max \{S: S \in B\}$. Then if $S<S^{c}$ we have $G\left(r_{e}, S\right)=\{0\}$ and if $S>S^{c}$ then $\left.G\left(r_{e}, S\right) \subset\right] 0,1[$.

Proof. From Proposition 2(i), $S^{c}>0$ since it must be greater than $\left(\frac{X}{r_{e}}\right)^{\frac{1}{\alpha_{e}}}$. From Lemma $3, S^{c}<+\infty$. 


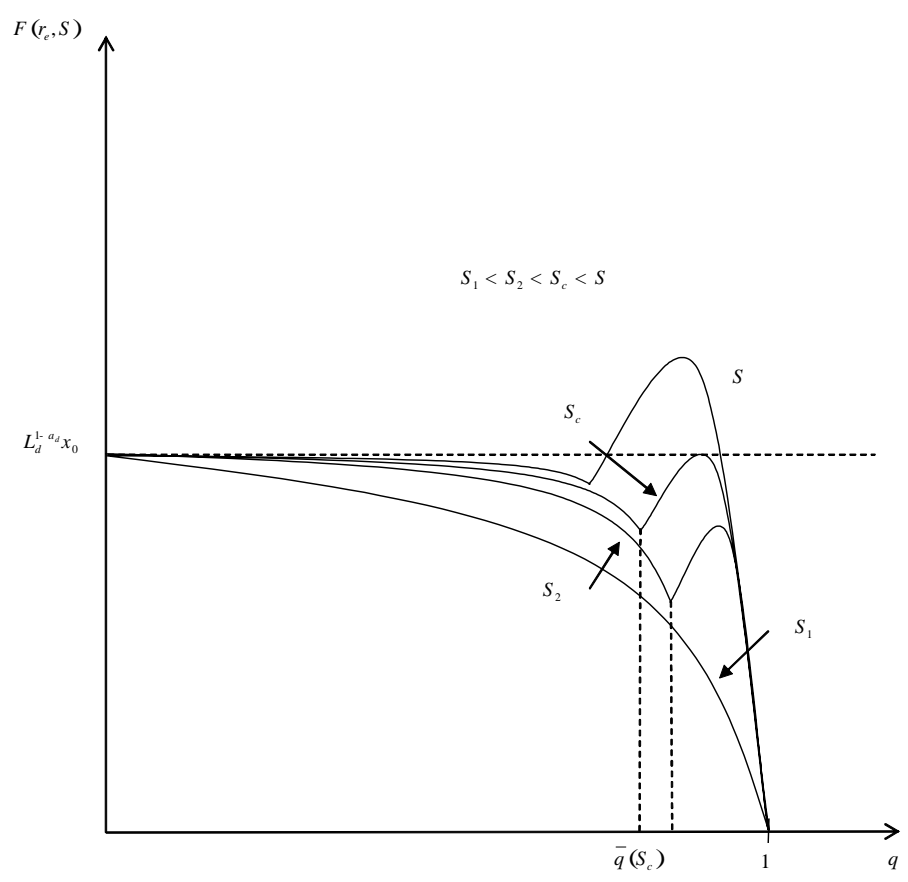

Figure 3: 
First, observe that if $S<S^{c}$ then $F\left(r_{e}, S\right)=x_{0} L_{d}^{*^{1-\alpha_{d}}}$. Indeed, we have

$$
\forall \theta \in[0,1], \psi\left(r_{e}, S, \theta\right) \leq \psi\left(r_{e}, S^{c}, \theta\right) .
$$

Hence $F\left(r_{e}, S\right)=\max _{\theta}\left\{\psi\left(r_{e}, S, \theta\right)\right\} \leq \max _{\theta}\left\{\psi\left(r_{e}, S^{c}, \theta\right)\right\}=F\left(r_{e}, S^{c}\right)=$ $x_{0} L_{d}^{*^{1-\alpha_{d}}}$. Since $\forall S \geq 0, F\left(r_{e}, S\right) \geq x_{0} L_{d}^{*^{1-\alpha} d}$, we have $F\left(r_{e}, S\right)=x_{0} L_{d}^{*^{1-\alpha}}$ Now, (i) if $S>S^{c}$, then from the very definition of $S^{c}$, we have $G\left(r_{e}, S\right) \subset$ ] $0,1[$.

(ii) If $S<S^{c}$, then take some $S_{0}<S^{c}$. Suppose for $S_{0}$ we have two solutions $\theta_{M}^{1}=0$ and $\theta_{M}^{2}>0$. There must be $\left.\bar{\theta}_{0} \in\right] 0,1\left[\right.$ which satisfies $r_{e} S_{0}^{\alpha_{e}}\left(\bar{\theta}_{0}\right)^{\alpha_{e}}=$ $X$ (if not, $\forall \theta, r_{e} S_{0}^{\alpha_{e}} \leq X$, and $G\left(r_{e}, S_{0}\right)=\{0\}$ ). For $\left.\left.\theta \in\right] 0, \bar{\theta}_{0}\right]$, we have $\psi\left(r_{e}, S, \theta\right)=(1-\theta)^{\alpha_{d}} x_{0} L_{d}^{*^{1-\alpha} d}<\psi\left(r_{e}, S, 0\right)=x_{0} L_{d}^{*^{1-\alpha} d}$. Hence $\theta_{M}^{2}>\bar{\theta}_{0}$. Let $S_{0}<S_{1}<S^{c}$, and $\bar{\theta}_{1}$ satisfy $r_{e} S_{1}^{\alpha_{e}} \bar{\theta}_{1}^{\alpha_{e}}=X$. Then $\theta_{M}^{2}>\bar{\theta}_{0}>\bar{\theta}_{1}$. We have

$$
\begin{gathered}
\phi\left(r_{e} S_{0}^{\alpha_{e}}\left(\theta_{M}^{2}\right)^{\alpha_{e}}\right)=x_{0}+\gamma\left(r_{e} S_{0}^{\alpha_{e}}\left(\theta_{M}^{2}\right)^{\alpha_{e}}\right) \\
\phi\left(r_{e} S_{1}^{\alpha_{e}}\left(\theta_{M}^{2}\right)^{\alpha_{e}}\right)=x_{0}+\gamma\left(r_{e} S_{1}^{\alpha_{e}}\left(\theta_{M}^{2}\right)^{\alpha_{e}}\right)>\phi\left(r_{e} S_{0}^{\alpha_{e}}\left(\theta_{M}^{2}\right)^{\alpha_{e}}\right) .
\end{gathered}
$$

We obtain a contradiction

$$
\begin{gathered}
x_{0} L_{d}^{*^{1-\alpha_{d}}}=F\left(r_{e}, S_{1}\right) \geq \phi\left(r_{e} S_{1}^{\alpha_{e}}\left(\theta_{M}^{2}\right)^{\alpha_{e}}\right)\left(1-\theta_{M}^{2}\right)^{\alpha_{d}} x_{0} L_{d}^{*^{1-\alpha_{d}}} \\
>\phi\left(r_{e} S_{0}^{\alpha_{e}}\left(\theta_{M}^{2}\right)^{\alpha_{e}}\right)\left(1-\theta_{M}^{2}\right)^{\alpha_{d}} x_{0} L_{d}^{*^{1-\alpha_{d}}}=F\left(r_{e}, S_{0}\right)=x_{0} L_{d}^{*^{1-\alpha_{d}}} .
\end{gathered}
$$

When the economy is subjected to an adoption effect, the impact of new technologies is not instantaneous on the TFP of consumption sector. Consequently, the production and the use of new technologies is highly dependent of the level of wealth of the economy, and it is not always optimal to use them. On the contrary, for a level of wealth lower than $S^{c}$, it is optimal for the developping country to devote all its ressources to physical capital accumulation.

\section{The Dynamic Model}

\subsection{The Model}

We consider now an economy with one infinitely lived representative agent who has an intertemporal utility function. She has the possibilty to consume or to save at each period $t$. Savings are directly used to buy an equivalent amount of capital. This capital as before can be of two kinds, technological 
or physical capital. As before, we suppose that the technological capital costs more than the physical capital. There is no change in the production functions of the consumption goods and of the new technology.

\subsubsection{The program}

The social planner will solve the following program.

$$
\max \sum_{t=0}^{+\infty} \beta^{t} u\left(c_{t}\right) \text { with } 0<\beta<1,
$$

under the constraints: for every date $t$,

$$
\begin{gathered}
c_{t}+S_{t+1} \leq \phi\left(Y_{e, t}\right) K_{d, t}^{\alpha_{d}} L_{d, t}^{\left(1-\alpha_{d}\right)}, \\
Y_{e, t}=A_{e} K_{e, t}^{\alpha_{e}} L_{e, t}^{1-\alpha_{e}}, \\
L_{d, t} \leq L_{d}^{*}, L_{e, t} \leq h L_{e}^{*}, \\
K_{d, t}+\lambda K_{e, t}=S_{t} .
\end{gathered}
$$

The initial resource $S_{0}>0$ is given.

This problem is equivalent to:

$$
\max \sum_{t=0}^{+\infty} \beta^{t} u\left(c_{t}\right)
$$

under the constraints: for every date $t$,

$$
\begin{gathered}
c_{t}+S_{t+1} \leq H\left(r_{e}, S_{t}\right) \text { with } \\
H\left(r_{e}, S_{t}\right)=\max _{\theta} \phi\left(r_{e} \theta^{\alpha_{e}} S_{t}^{\alpha_{e}}\right)(1-\theta)^{\alpha_{d}} L_{d}^{*^{1-\alpha_{d}}} S_{t}^{\alpha_{d}},
\end{gathered}
$$

and $S_{0}>0$ is given.

Recall that, as in the previous section, $r_{e}=\frac{A_{e} h^{1-\alpha_{e}} L_{e}^{1-\alpha_{e}}}{\lambda^{\alpha_{e}}}$.

We maintain the assumptions stated in Section 3 on $\phi$. We add for this section:

$\left(H_{1}\right)$ The function $u$ is strictly concave, strictly increasing and satisfies $u(0)=0$ and the Inada Condition $u^{\prime}(0)=+\infty$.

From the Maximum Theorem, $H$ is continuous. It is obviously strictly increasing with respect to $S$ and $H\left(r_{e}, 0\right)=0$.

From Proposition 4, there exists a critical value $S^{c}$ such that: 
(i) If $S<S^{c}$, then the set of optimal values $\theta_{M}, G\left(r_{e}, S\right)$, equals $\{0\}$, and

(ii) if $S>S^{c}$, then $\left.G\left(r_{e}, S\right) \subset\right] 0,1[$.

Since the utility function is strictly increasing, at the optimum the constraints will be binding.

$$
c_{t}=H\left(r_{e}, S_{t}\right)-S_{t+1}
$$

A sequence $\left(S_{t}\right)_{t=0 \ldots \infty}$ is called feasible from $S_{0} \geq 0$ if we have $\forall t, 0 \leq$ $S_{t+1} \leq H\left(r_{e}, S_{t}\right)$. Thus the initial program is equivalent to the following problem

$$
\max \sum_{t=0}^{\infty} \beta^{t} u\left(H\left(r_{e}, S_{t}\right)-S_{t+1}\right)
$$

under the constraints,

$$
0 \leq S_{t+1} \leq H\left(r_{e}, S_{t}\right), \text { for all } t \geq 0,
$$

with $S_{0}>0$ given. ${ }^{2}$.

\subsection{Properties of the optimal path}

In this subsection, we will give some properties of the optimal path of our economy. In particular, we will show it is monotonic and does not converge to 0 . Under some stronger conditions, we will show that any optimal path will grow without boundaries. Along this growth path, after a date $T$, the economy will use new technology to produce consumption goods.

Lemma 5 Every optimal path is monotonic

Proof. Notice that we have the following Bellman equation. Let $V$ be the value-function of the problem. We have

$$
\forall S_{0} \geq 0, V\left(S_{0}\right)=\max \left\{u\left(H\left(r_{e}, S_{0}\right)-S\right)+\beta V(S): 0 \leq S \leq H\left(r_{e}, S_{0}\right)\right\}
$$

Let $\Gamma$ denote the optimal correspondence. From Amir [1996], this correspondence is non decreasing, i.e., if $S_{0}^{\prime}<S_{0}$ then $\forall S_{1}^{\prime} \in \Gamma\left(r_{e}, S_{0}^{\prime}\right)$, and $\forall S_{1} \in \Gamma\left(r_{e}, S_{0}\right), S_{1}^{\prime} \leq S_{1}$. Hence, any optimal path must be monotonic.

\footnotetext{
${ }^{2}$ We assume that the utility function $u$ is such that the sum $\sum_{t=0}^{\infty} \beta^{t} u\left(H\left(r_{e}, S_{t}\right)-S_{t+1}\right)$ is real-valued for any feasible sequence $\left\{S_{t}\right\}$
} 
Proposition 6 Every optimal trajectory $\left(S_{t}^{*}\right)$ from $S_{0}>0$ cannot converge to 0 .

Proof. Suppose that $S_{t}^{*} \rightarrow 0$. Then for $t \geq T$, we have: $S_{t}^{*}<S^{c}$. Hence, $\forall t>T, H\left(r_{e}, S_{t}^{*}\right)=x_{0} L_{d}^{*^{1-\alpha_{d}}} S_{t}^{* \alpha_{d}}$ and $H_{S}^{\prime}\left(r_{e}, S_{t}^{*}\right) \rightarrow \infty$, because, $S_{t} \rightarrow 0$. As $u^{\prime}(0)=+\infty$, we have Euler equation for $t>T$,

$$
u^{\prime}\left(c_{t}^{*}\right)=\beta u^{\prime}\left(c_{t+1}^{*}\right) H_{S}^{\prime}\left(r_{e}, S_{t+1}^{*}\right) .
$$

There exists $T_{0} \geq T$ such that for all $t \geq T_{0}$ we have $H_{S}^{\prime}\left(r_{e}, S_{t+1}^{*}\right) \beta>1$. That implies $u^{\prime}\left(c_{t}^{*}\right)>u^{\prime}\left(c_{t+1}^{*}\right)$ or equivalently, $c_{t+1}^{*}>c_{t}^{*} \geq c_{T_{0}}^{*}>0$. That is contradictory with $S_{t}^{*} \rightarrow 0$ (because it would have for consequence $c_{t}^{*} \rightarrow 0$ ).

Let us denote by $K_{d, t}^{*}$ and $K_{e, t}^{*}$ respectively the optimal values of the physical and the technological capital stock and let $\theta_{t}^{*}$ the associated optimal capital share, i.e. $K_{d, t}^{*}=\left(1-\theta_{t}^{*}\right) S_{t}^{*}$ and $\lambda K_{e, t}^{*}=\theta_{t}^{*} S_{t}^{*}$.

Proposition 7 Let $S^{s}$ be defined by $x_{0} L_{d}^{*^{1-\alpha} d} \alpha_{d}\left(S^{s}\right)^{\alpha_{d}-1}=\frac{1}{\beta}$. Suppose $S^{s}>S^{c}$. Let $S_{0}>0$. Then there exists $T$ such that: $\forall t>T, G\left(r_{e}, S_{t}^{*}\right) \subset$ ]0,1[ or equivalently $K_{e, t}^{*}>0$.

Proof. When $S_{0} \geq S^{c}$, since the optimal path $\left(S_{t}^{*}\right)$ is nondecreasing, we have $\left.\forall t>0, G\left(r_{e}, S_{t}^{*}\right) \subset\right] 0,1\left[\right.$ or equivalently $K_{e, t}^{*}>0$.

Consider the case $S_{0}<S^{c}$. If for any $t, K_{e, t}^{*}=0$, then the optimal path $\left(S_{t}^{*}\right)$ will converge to $S^{s}$ (see e.g. Le Van and Dana, [2005], Chapter 2). Since we assume $S^{s}>S^{c}$, there will be $t$ with $S_{t}^{*}>S^{c}$. In this case $K_{e, t}^{*}>0$ which is contradictory.

So, let $T$ be the first date with $S_{T}^{*}>S^{c}$. Since the optimal path $\left(S_{t}^{*}\right)$ is nondecreasing, we will have $K_{e, t}^{*}>0$ for every $t>T$.

$S^{s}$ is the steady-state of our economy in the case of concave technology. If the critical value from which the economy becomes to import technological capital and to produce new technologies $\left(S^{c}\right)$ is higher than the steady-state value, the economy will never take off. In fact it will converge to its steadystate with a constant value of income per capita. On the contrary, if the steady-state value is higher than the critical wealth from which the economy produces new technologies, it will follow an endogenous growth path with a constant increase in income per capita. $S^{c}$ depends on the quantity of high-skilled labor and human capital initially available in the economy. Consequently, the model is able to explain different patterns of economic development according to the initial value of these variables. Moreover, it 


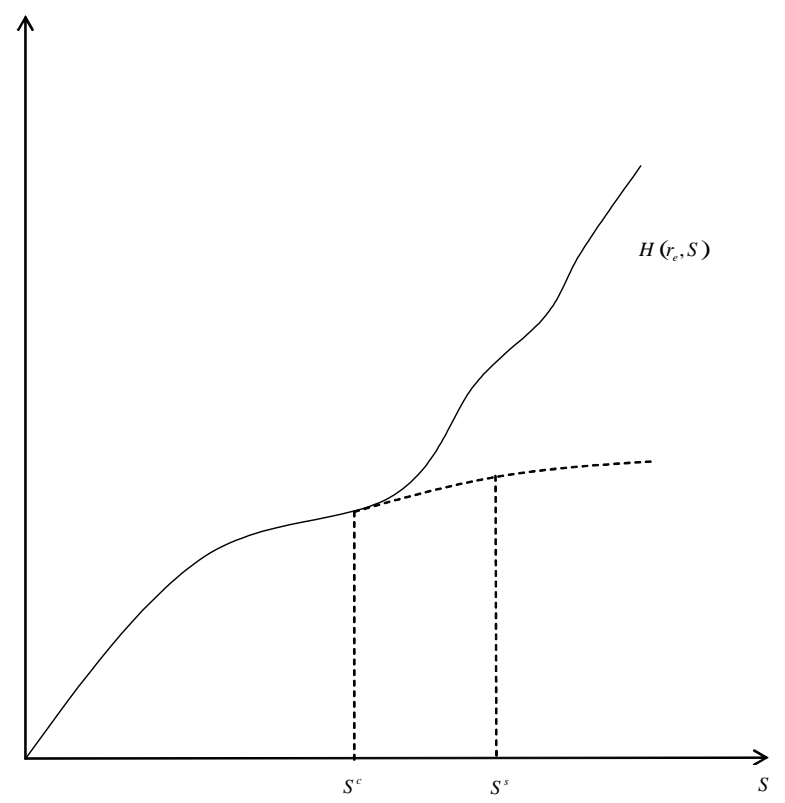

Figure 4:

is possible for a country to exhibit in the first stage of its development conditional convergence to its steady-state and after a certain period of time a complete divergence. Figure 4 gives a graphical interpretation of proposition 7.

We will show in the case of $S^{s}>S^{c}$ that the optimal path $S_{t}^{*}$ may converge to $+\infty$ with $\theta_{t}^{*} \rightarrow \theta^{\infty}=\frac{\alpha_{e}}{\alpha_{e}+\alpha_{d}}$. Thus, the optimal capital stocks converge also to $+\infty$.

We will now assume

$\left(H_{2}\right)$ The function $\phi$ has the following form

$$
\phi(x)=\left\{\begin{array}{c}
x_{0}, \forall x \leq X \\
x_{0}+a(x-X), \forall x \geq X \text { with } a>0 .
\end{array}\right\}
$$

Lemma 8 Assume $\left(H_{2}\right)$. (i) The function $F\left(r_{e}, S\right)$ is continuously differentiable with respect to $S$ in $] 0, S^{c}[\bigcup] S^{c},+\infty\left[\right.$. At $S^{c}$, it has left derivative (equal to 0 ) and right derivative.

(ii) For $S>S^{c}$, there exists a unique $\theta_{M}(S) \in G\left(r_{e}, S\right)$. Moreover, when $S$ converges to $+\infty$, then $\theta_{M}(S)$ converges to $\theta^{\infty}=\frac{\alpha_{e}}{\alpha_{e}+\alpha_{d}}$. 
Proof. (i) (a) When $S<S^{c}$, from Proposition ??, we have $F\left(r_{e}, S\right)=$ $x_{0} L_{d}^{* 1-\alpha_{d}}$.

(b) Consider the case where $S>S^{c}$. Let $\bar{\theta}(S)$ satisfy $r_{e} S^{\alpha_{e}} \bar{\theta}(S)^{\alpha_{e}}=X$.

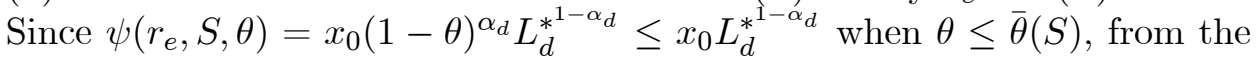
very definition of $S^{c}$, any solution must be larger than $\bar{\theta}(S)$. Thus, any solution $\theta$ must be interior to the interval $] \bar{\theta}(S), 1\left[\right.$, because $\psi\left(r_{e}, S, 1\right)=0$. The solution is unique since $\psi\left(r_{e}, S, \theta\right)$ is strongly concave in $\theta$. One can check that $\frac{\partial \psi}{\partial \theta}<0$. It satisfies $\psi_{\theta}^{\prime}\left(r_{e}, S, \theta\right)=0$. Tedious computations give:

$$
\frac{\alpha_{e}}{\alpha_{d}} \theta^{\alpha_{e}-1}(1-\theta)=\frac{x_{0}-a X}{a r_{e} S^{\alpha_{e}}}+\theta^{\alpha_{e}}
$$

The left side member is a decreasing function in $\theta$ while the right side one is increasing in $\theta$. The solution $\theta_{M}$ is unique. One can check that

$$
\frac{d \theta_{M}}{d S}=\frac{A}{B}
$$

with $A=\frac{a X-x_{0}}{a r_{e}} S^{\alpha_{e}-1}$ and $B=\frac{\alpha_{e}-1}{\alpha_{d}} \theta_{M}^{\alpha_{e}-2}-\left(1+\frac{\alpha_{e}}{\alpha_{d}}\right) \theta_{M}^{\alpha_{e}-1}<0$. Thus $F\left(r_{e}, \cdot\right)$ is differentiable for $S>S^{c}$.

(c) When $S=S^{c}$, there is a solution $\theta_{M}^{1}=0$ and another $\theta_{M}^{2}$ which is the unique solution to equation (14). From Clarke ( [1983], theorem 2.8.2), there is a right derivative equal to $\psi_{S}^{\prime}\left(r_{e}, S^{c}, \theta_{M}^{2}\right)$ and a left derivative which is trivially zero.

(ii) In (i) (b), we have shown that $G\left(r_{e}, S\right)$ is a singleton $\left\{\theta_{M}(S)\right\}$ when $S>$ $S^{c}$. Taking the limit when $S \rightarrow+\infty$, we obtain that $\theta_{M}(S) \rightarrow \theta^{\infty}=\frac{\alpha_{e}}{\alpha_{e}+\alpha_{d}}$.

Proposition 9 We maintain the same assumptions as in Lemma 8. We add

$\left(H_{3}\right) \alpha_{e}+\alpha_{d}>1$.

There exist $\bar{A}_{e}>0$ (or $\left.\bar{h}\right)$ such that if $A_{e}>\bar{A}_{e}($ or $h>\bar{h})$ then $K_{e, t}^{*} \rightarrow$ $+\infty$ and $\theta_{t}^{*} \rightarrow \theta^{\infty}=\frac{\alpha_{e}}{\alpha_{e}+\alpha_{d}}$.

Proof. It suffices to show that $H_{S}^{\prime}\left(r_{e}, S\right) \neq \frac{1}{\beta}, \forall S$.

Case 1: $x_{0}-a X<0$. In this case $\frac{d \theta_{M}(S)}{d S}<0$ when $S \geq S_{c}$ (see (15)). Therefore, for every $S \geq S_{c}, \theta_{M}(S)>\theta^{\infty}$.

Let $\theta_{M}\left(S^{c}\right)$ be the unique maximizer associated with $F\left(r_{e}, S^{c}\right)$ which is strictly positive. For short, write $\theta_{c}$ instead of $\theta_{M}\left(S^{c}\right)$. Then $\left(\theta_{c}, S^{c}\right)$ satisfy (14) and $F\left(r_{e}, S^{c}\right)=x_{0} L^{*^{1-\alpha_{d}}}$. We obtain:

$$
\left(x_{0}-a X\right) \frac{\alpha_{e} \theta_{c}^{\alpha_{e}-1}\left(1-\theta_{c}\right)^{\alpha_{d}+1}}{\left(\alpha_{e}+\alpha_{d}\right) \theta_{c}^{\alpha_{e}}-\alpha_{e} \theta_{c}^{\alpha_{e}-1}}=x_{0} .
$$


We see that $\theta_{c}$ is independent of $r_{e}$ hence $A_{e}$ and $h$. If $A_{e}$ (or $h$ ) is large enough, then from (14) $S^{c}<S^{s}=\left(\beta x_{0} \alpha_{d}\right)^{\frac{1}{1-\alpha_{d}}}$ and $H_{S}^{\prime}\left(r_{e}, S\right) \neq \frac{1}{\beta}, \forall S \leq S^{c}$. Consider the case where $S>S^{c}$. From the envelope theorem and relation 14, we have

$$
H_{S}^{\prime}\left(r_{e}, S\right)=a r_{e} \alpha_{e} \theta_{M}(S)^{\alpha_{e}-1}\left(1-\theta_{M}(S)\right)^{\alpha_{d}} S^{\alpha_{e}+\alpha_{d}-1} .
$$

We can bound this derivative from below:

$$
H_{S}^{\prime}\left(r_{e}, S\right)>a r_{e} \alpha_{e}\left(1-\theta_{c}\right)^{\alpha_{d}} S^{\alpha_{e}+\alpha_{d}-1}
$$

and hence

$$
S<\left(\frac{1}{\beta a r_{e} \alpha_{e}\left(1-\theta_{c}\right)^{\alpha_{d}}}\right)^{\frac{1}{\alpha_{e}+\alpha_{d}-1}}, \text { if } H_{S}^{\prime}\left(r_{e}, S\right)=\frac{1}{\beta} .
$$

Again from (14) we can write $S^{c}=\left(\frac{\zeta\left(a, x_{0}, X\right)}{a_{e}}\right)^{\frac{1}{\alpha_{e}}}$ where the function $\zeta$ can be easily computed. One can also easily check that if $A_{e}$ (hence $r_{e}$ ) is sufficiently large then $S$ will be less than $S^{c}$ which is a contradiction.

Case 2: $x_{0}-a X>0$. As above, $\theta_{c}$ is independent of $r_{e}$. If $A_{e}$ (or $h$ ) is large enough, then from (14) $S^{c}<S^{s}=\left(\beta x_{0} \alpha_{d}\right)^{\frac{1}{1-\alpha_{d}}}$ and $H_{S}^{\prime}\left(r_{e}, S\right) \neq \frac{1}{\beta}, \forall S \leq S^{c}$. When $S>S^{c}$, (14), we have $\theta_{M}(S)<\theta^{\infty}$. We then have

$$
H_{S}^{\prime}\left(r_{e}, S\right)>a r_{e} \alpha_{e}\left(1-\theta^{\infty}\right)^{\alpha_{d}} S^{\alpha_{e}+\alpha_{d}-1}
$$

and hence

$$
S<\left(\frac{1}{\beta a r_{e} \alpha_{e}\left(1-\theta_{c}\right)^{\alpha_{d}}}\right)^{\frac{1}{\alpha_{e}+\alpha_{d}-1}}, \text { if } H_{S}^{\prime}\left(r_{e}, S\right)=\frac{1}{\beta} .
$$

Apply the same argument as above to obtain a contradiction.

Case 3: $x_{0}=a X$. From (14), we have $\theta_{M}(S)=\theta^{\infty}, \forall S \geq S^{c}$. It is easy to check that

$$
S^{c_{e}^{\alpha}} a r_{e} \theta^{\infty_{e}^{\alpha}}\left(1-\theta^{\infty}\right)^{\alpha_{d}}=x_{0}
$$

Obviously, when $A_{e}$ or $h$ are large then $S^{c}<S^{s}$. Since we now have for $S>S^{c}$,

$$
H\left(r_{e}, S\right)=a r_{e} \theta^{\infty_{e}^{\alpha}}\left(1-\theta^{\infty}\right)^{\alpha_{d}} L^{*^{\left(1-\alpha_{d}\right)}} S^{\alpha_{e}+\alpha_{d}}
$$

we get

$$
H_{S}^{\prime}\left(r_{e}, S\right)=a r_{e} \theta^{\infty_{e}^{\alpha}}\left(1-\theta^{\infty}\right)^{\alpha_{d}} L^{*^{\left(1-\alpha_{d}\right)}}\left(\alpha_{e}+\alpha_{d}\right) S^{\alpha_{e}+\alpha_{d}-1}
$$

and $S<S^{c}$ if $H_{S}^{\prime}\left(r_{e}, S\right)=\frac{1}{\beta}$. That is a contradiction. The proof is complete. 


\section{Mobility of labour}

We now assume that high-skilled people can work in the sector of consumption good if the demand for high-skilled labor is not sufficient in the research sector. But the reverse is not possible, i.e. low-skilled people cannot move in the new technology sector. We therefore replace the constraints labor demands $(5,6)$ by

$$
L_{d} \leq L_{e}^{*}+L_{d}^{*}
$$

and

$$
L_{e} \leq h L_{e}^{*}
$$

We can write $L_{e}=\mu h L_{e}^{*}, L_{d}=L_{d}^{*}+(1-\mu) L_{e}^{*}$ with $\mu \in[0,1]$. We assume that when the high-skilled workers are in the consumption sector their human capital equals the one of this sector.

The production function in the new technology sector will be:

$$
Y_{e}=\frac{A_{e}}{\lambda^{\alpha_{e}}} \theta^{\alpha_{e}} S^{\alpha_{e}} \mu^{1-\alpha_{e}} h^{1-\alpha_{e}} L_{e}^{*^{1-\alpha_{e}}}
$$

where $\mu$ represents the part of high skilled labor used in this sector.

The production function in the consumption good sector now is:

$$
Y_{d}=\phi\left(Y_{e}\right)(1-\theta)^{\alpha_{d}} S^{\alpha_{d}}\left(L_{d}^{*}+(1-\mu) L_{e}^{*}\right)^{1-\alpha_{d}}
$$

\subsection{The one period model}

Let $r_{e}=\frac{A_{e}}{\lambda^{\alpha_{e}}} h^{1-\alpha_{e}} L_{e}^{*^{1-\alpha_{e}}}$. The program of the social planner is:

$$
\begin{aligned}
& \max _{0 \leq \theta \leq 1} Y_{d}=\phi\left(r_{e} \theta^{\alpha_{e}} S^{\alpha_{e}} \mu^{1-\alpha_{e}}\right)(1-\theta)^{\alpha_{d}} S^{\alpha_{d}}\left(L_{d}^{*}+(1-\mu) L_{e}^{*}\right)^{1-\alpha_{d}} . \\
& 0 \leq \mu \leq 1
\end{aligned}
$$

Let

$$
\varphi\left(r_{e}, S, \theta, \mu\right)=\phi\left(r_{e} \theta^{\alpha_{e}} S^{\alpha_{e}} \mu^{1-\alpha_{e}}\right)(1-\theta)^{\alpha_{d}}\left(L_{d}^{*}+(1-\mu) L_{e}^{*}\right)^{1-\alpha_{d}} .
$$

The problem is equivalent to

$$
\max _{(\theta, \mu) \in[0,1] \times[0,1]} \varphi\left(r_{e}, S, \theta, \mu\right) .
$$

Let

$$
F\left(r_{e}, S\right)=\max _{(\theta, \mu) \in[0,1] \times[0,1]} \varphi\left(r_{e}, S, \theta, \mu\right)
$$


Then $F\left(r_{e}, S\right) \geq x_{0}\left(L_{d}^{*}+L_{e}^{*}\right)^{1-\alpha_{d}}$. As before, define $B=\left\{S \geq 0: F\left(r_{e}, S\right)=\right.$ $\left.x_{0}\left(L_{d}^{*}+L_{e}^{*}\right)^{1-\alpha_{d}}\right\}$. It is easy to check that $B$ is compact and nonempty. The critical value is

$$
S^{c}=\max \{S: S \in B\}
$$

Observe that for $S>S^{c}$ the function

$$
Z\left(r_{e}, S, \theta, \mu\right)=\log \left(\varphi\left({ }_{r} e, S, \theta, \mu\right)\right)
$$

is strongly concave in $(\theta, \mu)$. Since to maximize $\varphi\left(r_{e}, S, \theta, \mu\right)$ is equivalent to maximize $Z\left(r_{e}, S, \theta, \mu\right)$ when $S>S^{c}$, the solution $\left(\theta_{M}(S), \mu_{M}(S)\right)$ will be unique. Obviously, if $S>S^{c}$ then $\theta_{M}(S)>0$ (if not, we will have $\mu_{M}(S)=0$ and $\left.F\left(r_{e}, S\right)=x_{0}\left(L_{d}^{*}+L_{e}^{*}\right)^{1-\alpha_{d}}\right)$. We have the following result

Proposition 10 Assume $\frac{L_{e}^{*}}{L_{d}^{*}}<\frac{1-\alpha_{e}}{1-\alpha_{d}}$. Then there exists $\bar{S}$ such that if $S>$ $\bar{S}$ then $\mu_{M}(S)=1$.

Proof. Assume the statement false. Then there exists a sequence $\left(S_{n}\right)$ converging to $+\infty$ with $\mu_{M}\left(S_{n}\right)<1, \forall n$. We may assume $\mu_{M}\left(S_{n}\right) \rightarrow \bar{\mu} \leq 1$ and $\theta_{M}\left(S_{n}\right) \rightarrow \bar{\theta}$. For short, write $\mu_{n}=\mu_{M}\left(S_{n}\right), \theta_{n}=\theta_{M}\left(S_{n}\right)$. For every $n$, we have

$$
\begin{aligned}
& x_{0}+a\left(r_{e} S_{n}^{\alpha_{e}} \theta_{n}^{\alpha_{e}} \mu_{n}^{1-\alpha_{e}}-X\right)\left(1-\theta_{n}\right)^{\alpha_{d}}\left(L_{d}^{*}+\left(1-\mu_{n}\right) L_{e}^{*}\right)^{1-\alpha_{d}} \\
& \geq x_{0}+a\left(r_{e} S_{n}^{\alpha_{e}} \theta^{\alpha_{e}} \mu^{1-\alpha_{e}}-X\right)(1-\theta)^{\alpha_{d}}\left(L_{d}^{*}+(1-\mu) L_{e}^{*}\right)^{1-\alpha_{d}}
\end{aligned}
$$

for every $\theta \in[0,1]$, every $\mu \in[0,1]$. This inequality is equivalent to

$$
\begin{aligned}
& \quad \frac{x_{0}}{S_{n}^{\alpha_{e}}}+a\left(r_{e} \theta_{n}^{\alpha_{e}} \mu_{n}^{1-\alpha_{e}}-\frac{X}{S_{n}^{\alpha_{e}}}\right)\left(1-\theta_{n}\right)^{\alpha_{d}}\left(L_{d}^{*}+\left(1-\mu_{n}\right) L_{e}^{*}\right)^{1-\alpha_{d}} \\
& \geq \frac{x_{0}}{S_{n}^{\alpha_{e}}}+a\left(r_{e} \theta^{\alpha_{e}} \mu^{1-\alpha_{e}}-\frac{X}{S_{n}^{\alpha_{e}}}\right)(1-\theta)^{\alpha_{d}}\left(L_{d}^{*}+(1-\mu) L_{e}^{*}\right)^{1-\alpha_{d}} .
\end{aligned}
$$

Let $S_{n}$ converge to infinity. We obtain

$$
\begin{gathered}
a r_{e} \bar{\theta}^{\alpha_{e}} \bar{\mu}^{1-\alpha_{e}}(1-\bar{\theta})^{\alpha_{d}}\left(L_{d}^{*}+(1-\bar{\mu}) L_{e}^{*}\right)^{1-\alpha_{d}} \\
\left.\geq a r_{e} \theta^{\alpha_{e}} \mu^{1-\alpha_{e}}(1-\theta)^{\alpha_{d}}\left(L_{d}^{*}+(1-\mu) L_{e}^{*}\right)^{1-\alpha_{d}}>0 \text { if } \theta \in\right] 0,1[, \mu>0 .
\end{gathered}
$$

That implies $\bar{\theta} \in] 0,1[, \bar{\mu}>0$. But for every $n$ we also have:

$$
\begin{aligned}
& x_{0}+a\left(r_{e} S_{n}^{\alpha_{e}} \theta_{n}^{\alpha_{e}} \mu_{n}^{1-\alpha_{e}}-X\right)\left(1-\theta_{n}\right)^{\alpha_{d}}\left(L_{d}^{*}+\left(1-\mu_{n}\right) L_{e}^{*}\right)^{1-\alpha_{d}} \\
\geq & x_{0}+a\left(r_{e} S_{n}^{\alpha_{e}} \theta_{n}^{\alpha_{e}} \mu^{1-\alpha_{e}}-X\right)\left(1-\theta_{n}\right)^{\alpha_{d}}\left(L_{d}^{*}+(1-\mu) L_{e}^{*}\right)^{1-\alpha_{d}} .
\end{aligned}
$$


Since $\mu_{n} \in(0,1)$ we get the first order condition for $\mu_{n}$ :

$$
\begin{gathered}
\operatorname{ar}_{e} \theta_{n}^{\alpha_{e}}\left(1-\alpha_{e}\right) \mu_{n}^{-\alpha_{e}}\left(L_{d}^{*}+(1-\mu) L_{e}^{*}\right)= \\
L_{e}^{*}\left(1-\alpha_{d}\right)\left[\frac{x_{0}-a X}{S_{n}}+a r_{e} \theta_{n}^{\alpha_{e}} \mu_{n}^{1-\alpha_{e}}\right] .
\end{gathered}
$$

Let $S_{n}$ converge to infinity. We obtain $\bar{\mu}=\frac{\left(1-\alpha_{e}\right)\left(L_{d}^{*}+L_{e}^{*}\right)}{\left(2-\alpha_{e}-\alpha_{d}\right) L_{e}^{*}}$. And $\bar{\mu}>1$ if $\frac{L_{e}^{*}}{L_{d}^{*}}<\frac{1-\alpha_{e}}{1-\alpha_{d}}$. That implies $\mu_{n}=1$ for any $n$ large enough.

Proposition 11 Let $S>S^{c}$. Assume $\frac{L_{e}^{*}}{L_{d}^{*}}<\frac{1-\alpha_{e}}{1-\alpha_{d}}$ and $x_{0}-a X \leq 0$. Then $\mu_{M}(S)=1$.

Proof. To make short, write $\left(\theta_{M}, \mu_{M}\right)$ instead of $\left(\theta_{M}(S), \mu_{M}(S)\right)$. If $\left(\theta_{M}, \mu_{M}\right)$ are interior we have the following first-order conditions

$$
\theta_{M}^{\alpha_{e}-1} \mu_{M}^{1-\alpha_{e}} \alpha_{e}\left(1-\theta_{M}\right)=\left[\frac{x_{0}-a X}{a r_{e} S^{\alpha_{e}}}+\theta_{M}^{\alpha_{e}} \mu_{M}^{1-\alpha_{e}}\right] \alpha_{D}
$$

$\theta_{M}^{\alpha_{e}} \mu_{M}^{-\alpha_{e}}\left(1-\alpha_{e}\right)\left(L_{d}^{*}+\left(1-\mu_{M}\right) L_{e}^{*}\right)=\left[\frac{x_{0}-a X}{a r_{e} S^{\alpha_{e}}}+\theta_{M}^{\alpha_{e}} \mu_{M}^{1-\alpha_{e}}\right]\left(1-\alpha_{d}\right) L_{e}^{*}(19$

If $x_{0}-a X \leq 0$, then from (19) we obtain $\mu_{M} \geq \mu^{\infty}=\frac{\left(1-\alpha_{e}\right)\left(L_{d}^{*}+L_{e}^{*}\right)}{\left(2-\alpha_{d}-\alpha_{e}\right) L_{e}^{*}}$. But if $\frac{L_{e}^{*}}{L_{d}^{*}}<\frac{1-\alpha_{e}}{1-\alpha_{d}}$, then $\mu^{\infty}>1$ and we have a contradiction. Since $\mu_{M}>0$, we must have $\mu_{M}=1$.

\subsection{The Dynamic Model}

Define

$$
L\left(r_{e}, S\right)=F\left(r_{e}, S\right) S^{\alpha_{d}} .
$$

The function $L$ is strictly increasing in $S$, continuous and $L\left(r_{e}, 0\right)=0$.

As before the optimal growth model is:

$$
\max \sum_{t=0}^{+\infty} \beta^{t} u\left(L\left(r_{e}, S_{t}\right)-S_{t+1}\right)
$$

under the constraints

$$
0 \leq S_{t+1} \leq L\left(r_{e}, S_{t}\right) S_{0}>0 \text { is given. }
$$

As in the previous dynamic model, from Amir (1996), the optimal path $\left(S_{t}^{*}\right)$ is monotonic.

We have the following proposition 
Proposition 12 Let $S^{s}$ satisfy

$$
\alpha_{d}\left(S^{s}\right)^{\alpha_{d}-1} x_{0}\left(L_{e}^{*}+L_{d}^{*}\right)^{1-\alpha_{d}}=\frac{1}{\beta} .
$$

Assume $S^{s}>S^{c}$. Then any optimal path $\left(S_{t}^{*}\right)$ from $S_{0}>0$ cannot converge to zero. Moreover, there will be a date $T$ such that, for all $t>T$, the optimal technological capital stock satisfies $K_{e, t}^{*}>0$.

Proof. If the optimal path $\left(S_{t}^{*}\right)$ converges to zero, then for all $t$ large enough, we have $S_{t}^{*}<S_{c}$. That implies the optimal values $\theta_{t}^{*}$ and $\mu_{t}^{*}$ are respectively 0 and 1 . To end the proof, see the proofs of Propositions 6, 7 .

Observe that along an optimal path, we cannot ensure the supply of highskilled labor supply be exhausted. The following proposition gives a condition for which that will be true.

Proposition 13 Assume that the optimal path $\left(S_{t}^{*}\right)$ converges to $+\infty$ and $\frac{L_{e}^{*}}{L_{d}^{*}}<\frac{1-\alpha_{e}}{1-\alpha_{d}}$. Then there exists $T$ such that $\forall t>T, \mu_{t}^{*}=1$.

Proof. That is a corollary of Proposition 10

Finally we have the following proposition.

Proposition 14 (i) Assume $\left(H_{3}\right)$. Then there exists $\bar{A}_{e}>0$ (or $\left.\bar{h}\right)$ such that if $a=\bar{a}, A_{e}>\bar{A}_{e}\left(\right.$ or $h>\bar{h}$ ) then $K_{e, t}^{*} \rightarrow+\infty$ and $\theta_{t}^{*} \rightarrow \theta^{\infty}=\frac{\alpha_{e}}{\alpha_{e}+\alpha_{d}}$. (ii) Moreover, if $\frac{L_{e}^{*}}{L_{d}^{*}}<\frac{1-\alpha_{e}}{1-\alpha_{d}}$, then there exists $T \geq 0$, such that $\mu_{t}^{*}=1$ for every $t \geq T$.

Proof. (i) The proof is similar to the one of Proposition 9.

(ii) When $x_{0}-a X \leq 0$ then from Proposition 11, we have $\mu_{t}^{*}=1$ for every $t \geq 0$. When $x_{0}-a X>0$, apply Proposition 13 .

Remark 15 Consider the case where $x_{0}=a X$ and $\alpha_{e}+\alpha_{d}>1$. From the first order conditions $(18,19)$ when $S>S^{c}$, we obtain that the optimal values $\left(\theta^{*}, \mu^{*}\right)$ are independent of $S$ and respectively equal

$$
\theta^{\infty}=\frac{\alpha_{e}}{\alpha_{e}+\alpha_{d}}, \mu^{\infty}=\frac{\left(1-\alpha_{e}\right)\left(L_{d}^{*}+L_{e}^{*}\right)}{\left(2-\alpha_{d}-\alpha_{e}\right) L_{e}^{*}} .
$$

The technological function of the optimal growth model becomes

$$
L\left(r_{e}, S\right)=x_{0}\left(L_{d}^{*}+L_{e}^{*}\right)^{1-\alpha_{d}} S^{\alpha_{d}} \text { if } S \leq S^{c},
$$


and

$L\left(r_{e}, S\right)=a r_{e} \theta^{\infty^{\alpha_{e}}}\left(1-\theta^{\infty}\right)^{\alpha_{d}} \mu^{\infty^{1-\alpha_{e}}}\left(L_{d}^{*}+\left(1-\mu^{\infty}\right) L_{e}^{*}\right)^{1-\alpha_{d}} S^{\alpha_{e}+\alpha_{d}-1}$, if $S>S^{c}$.

The function $L\left(r_{e}, \cdot\right)$ is concave-convex and differs from the case in Dechert and Nishimura (1983) where the technology is convex-concave.

\section{Conclusion}

We summarize the results we obtain in this paper.

1. When there is an adoption effect of new technologies by firms, there exists a critical value $S^{c}$ for the domestic resource. Below this value, it is not optimal for the country to invest in new technology. Above this value, it is optimal to invest in new technology. The critical value decreases when the human capital or/and the productivity of the new technology sector is high, or/and the price of the new technology capital is low.

2. When the human capital is high and/or the productivity of the new technology sector is high, in the dynamic setting, there is a date $T_{1}$ such that for any date beyond $T_{1}$ the country will adopt the new technology and grows without bound.

3. If we allow the high skilled workers to move to the consumption sector, then may be a date $T_{2}$ such that the new technology sector will use all the high skilled workers only after this date. This result shows that is not always optimal to have a very large number of high-skilled workers.

4. Here we want to sketch a country which faces the corruption phenomenon. There are many ways to formalize this phenomenon. One is to assume that the production function in the consumption sector exhibits fixed costs. But it will raise many mathematical complications. So, we use the way proposed by Dimaria and Le Van (2002). We assume that at every date $t$, the bribers divert a fraction $\eta \in(0,1)$ of national resource, $S_{t+1}$, devoted to the next period investment. The amount $\eta S_{t+1}$ either goes abroad or is used for consuming imported goods. In this case the constraints at every period are:

$$
c_{t}+S_{t+1}=L\left(r_{e},(1-\eta) S_{t}\right)
$$

and

$$
K_{d, t}+\lambda K_{e, t}=(1-\eta) S_{t}
$$

It is obvious that the new critical value $S^{\prime^{c}}$ equals $\frac{S^{c}}{1-\eta}$, i.e. larger than $S^{c}$. It converges to $+\infty$ when $\eta$ goes to 1 . The new value $S^{\prime s}$ (corresponding to the steady state of the optimal growth model without new technology) will be 
$S^{s}(1-\eta)^{\frac{\alpha_{d}}{1-\alpha_{d}}}$, i.e. smaller than $S^{s}$. It converges to zero when $\eta$ converges to one. Assume the initial wealth $S_{0}$ satisfy $S^{c}<S_{0}<S^{s}$ allowing the country to take off. In presence of high corruption ( $\eta$ close to 1 ), we have $S^{\prime s}<S_{0}<S^{\prime c}$ and the country will fall down by converging to $S^{\prime^{s}}$.

Observe that in this paper we obtain an optimal path which grows without bound in contrast with usual Ramsey models. Our condition is the same as in Kamihigashi and Roy (2005) for a more general setting.

\section{References}

[1] Amir, R., Sensitivity Analysis in Multisector Optimal Economic Models, Journal of Mathematical Economics, 25, pp. 123-141, 1996.

[2] Barro, R. Determinants of Economic Growth. A Cross-Country Empirical Study. MIT Press, Cambridge, 1997.

[3] Barro, R. and Sala-i-Martin, X., Economic Growth, McGraw Hill, New York, 1995.

[4] Benhabib, J. and Spiegel, M.M., The Role of Human Capital in Economic Development: Evidence from Aggregate Cross-Country Data, Journal of Monetary Economics, 34,2, 1994.

[5] Cass, D., Optimal Growth in an Aggregative Model of Capital Accumulation, Review of Economic Studies, 32, 1965.

[6] Ciccone, A and Matsuyama, K., Efficiency and Equilibrium with Dynamic Increasing returns due to Demand Complementarities, Econometrica, 67,2, 1999.

[7] Clarke ,F.H., Optimization and Nonsmooth Analysis, John Wiley and Sons, 1983.

[8] Dechert, W.D. and Nishimura, K., A Complete Characterization of Optimal Growth Paths in an Aggregated Model with a Non-Concave Production Function, Journal of Economic Theory, 31, pp. 332-354,1983.

[9] Dimaria, Ch-H. and Le Van, C., Optimal Growth, Debt, Corruption and R-D, Macroeconomic Dynamics, 6, pp. 597-613, 2002.

[10] Kamihigashi, T., and Roy, S., A Nonsmooth, Nonconvex Model of Optimal Growth, 2005 (to appear in Journal of Economic Theory). 
[11] Kim, J., and Lau, L., The Sources of Economic Growth in the East Asian Newly Industrial Countries, Journal of Japanese and International Economics, 8, 1994.

[12] Le Van, C. and Dana, R.A., Dynamic Programming in Economics, Kluwer Academic Publishers, 2003.

[13] Le Van, C. and Cagri Saglam, H., Quality of Knowledge Technology, Returns to Production Technology, and Economic Development, Macroeconomic Dynamics, 8, 147-161, 2004. 